\title{
Optical delivery of multiple opsin-encoding genes leads to targeted expression and white-light activation
}

\author{
Kamal Dhakal $^{1, \dagger}$, Subrata Batabyal ${ }^{1, \dagger}$, Weldon Wright ${ }^{2}$, Young-tae Kim ${ }^{3}$ and Samarendra Mohanty ${ }^{1,2}$
}

In photodegenerative diseases such as retinitis pigmentosa (RP) and age-related macular degeneration (AMD), progressive loss of vision occurs as a result of degeneration of the periphery of the retina and the macula, respectively. Current optogenetic stimulation-based approaches to vision restoration offer the advantages of cellular specificity, high resolution, and minimal invasiveness over electrode arrays; however, the clinical translation of optogenetic activation suffers from the lack of a method for the delivery of opsins into spatially targeted regions of a retina that has degenerated. Non-targeted opsin delivery through viral or non-viral methods to non-photodegenerated retinal areas will perturb these already functioning retinal regions. Furthermore, viral methods are subject to limitations on the delivery of large plasmids, such as fusion constructs of multiple spectrally separated opsins (e.g., channelrhodopsin-2 (ChR2), chimeric opsin variants (C1V1), ReaChR), which can provide higher photo-excitability than can a single narrow-band opsin under ambient light conditions. Here, we report the ultrafast near-infrared laser-based spatially targeted transfection of single and multiple opsins and present a comparison with the opsin expression distribution achieved using another non-viral, but non-targeted, transfection method, lipofection. Functional evaluation of cells transfected with multiple opsins using the laser method revealed a significantly higher white-light-induced photocurrent than in cells expressing a single opsin (ChR2). The laser-assisted targeted delivery of multiple opsin-encoding genes to the peripheral retina/macula is ideal for sensitizing retinal areas that have degenerated, thus paving the way toward the restoration of lost vision in RP/AMD patients.

Light: Science \& Applications (2015) 4, e352; doi:10.1038/Isa.2015.125; published online 6 November 2015

Keywords: gene delivery; optogenetics; optoporation; vision restoration

\section{INTRODUCTION}

The current methods for the optogenetic restoration of vision include the use of either channelrhodopsin-2 (ChR2) for non-specific stimulation of the retina ${ }^{1}$ or promoter-specific approaches such as those using Thy1 for targeting retinal ganglion cells (RGCs) ${ }^{2-4}$ and mGluR6 for targeting $\mathrm{ON}$ bipolar cells ${ }^{5}$. Furthermore, the expression of chloride-channel opsin (halorhodopsin, $\mathrm{NpHR}^{6}$ ) in longer-persisting cone photoreceptors has shown promise for vision restoration ${ }^{6}$. The optogenetic activation method is very promising because it requires only a low intensity of light, which can be delivered by a light-emitting diode or laser ${ }^{7-10}$. In addition, optogenetic approaches offer several advantages over electrical stimulation, such as cellular specificity, high temporal and spatial resolution, and minimal invasiveness ${ }^{7-8,11-14}$. However, the clinical translation of optogenetic activation for vision restoration suffers from the lack of a method for the delivery of opsinencoding genes into spatially targeted regions of a retina that has degenerated (e.g., the periphery of the retina in retinitis pigmentosa (RP) or the macula in age-related macular degeneration (AMD). Nontargeted opsin delivery through viral or non-viral methods to residual (non-photodegenerated) retinal areas will cause complications in the functioning of these areas that have not degenerated by interfering with the light-evoked potential in multiple layers of the retinal circuitry.

Recently, there has been considerable interest in the optical transfection of cells using ultrafast pulsed light ${ }^{15-23}$ because this approach offers selective targeting and higher efficiency and viability ( $>90 \%$ reported in vitro ${ }^{22}$ ) than other methods. Furthermore, femtosecond (fs) near-infrared (NIR) laser-based transfection has been shown to be safe, resulting in high efficiency and high survival rates $(93 \%)$ of optoporated embryos during development ${ }^{20}$, as well as suitable for in vivo gene delivery ${ }^{24}$. We have demonstrated ${ }^{25}$ the use of an ultrafast NIR laser microbeam for the optical delivery of opsin (ChR2)-yellow fluorescent protein (YFP) plasmids to spatially localized regions of the retina. Because non-linear interaction between the ultrafast laser beam and the cells occurs only within $1 \mu \mathrm{m}$ of the focus, only the ganglion cell layer (which is proximal to the laser beam) or even only a single RGC can be sensitized to light. However, to achieve higher cell sensitivity to ambient broadband (white) light, it is advantageous to deliver multiple, spectrally separated opsins into the targeted cells. The delivery of large fusion constructs of multiple spectrally separated opsins (e.g., ChR2, chimeric opsin variants (C1V1), ReaChR) is challenging for the currently achievable safe viral methods. The sepa-

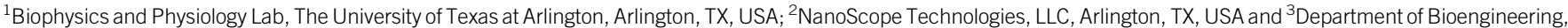
The University of Texas at Arlington, Arlington, TX, USA

†These authors contributed equally to this work

Correspondence: S Mohanty, Email: smohanty@uta.edu

Received 23 December 2014; revised 30 June 2015; accepted 4 July 2015; accepted article preview online 7 July 2015 
rate delivery of different opsins by multiple viral vectors can lead to immune reactions and may cause unwanted transfection of non-targeted tissue. Although several non-viral approaches, such as electroporation and lipofection, exist, these methods suffer from low transfection efficiency, loss of cell viability, and non-targeted transfection, similar to viral methods. Ultrafast NIR laser-mediated optopora$\operatorname{tion}^{23,25}$ can address these issues and holds promise for the delivery of large multi-opsin constructs via targeted transfection with single-cell resolution and high efficiency.

Herein, we report a comparative study of lipofection vs. the laser transfection of both single and multiple opsins. The fs laser transfection method was found to be capable of delivering large $(>10 \mathrm{~kb})$ multi-opsin constructs. The membrane-to-intracellular expression ratio was found to be higher in the case of laser transfection than lipofection because of less perturbation of cellular morphology. A higher photocurrent was observed in the case of HEK293 cells transfected with multiple opsins than in ChR2-sensitized HEK293 cells under white-light stimulation. The application of the spatially targeted laser transfection of multiple opsins in retinas that have degenerated will allow stimulation of the RGCs by broadband white light, leading to vision restoration in RP and AMD patients.

\section{MATERIALS AND METHODS}

\section{Opsin-encoding plasmid preparation}

The opsin-encoding plasmids were eluted with nuclease-free water upon receipt and stored at $-20{ }^{\circ} \mathrm{C}$. For transformation, $1 \mu \mathrm{L}$ of the DNA (10 ng) was added to a vial of JM109 (competent Escherichia coli) cells and then was mixed gently. The mixture-containing vials were incubated on ice for $30 \mathrm{~min}$, and the cells were subjected to heat shock for $60 \mathrm{~s}$ at $42{ }^{\circ} \mathrm{C}$ without shaking. The vials were then removed from the $42{ }^{\circ} \mathrm{C}$ bath and placed on ice for $2 \mathrm{~min}$, and $250 \mu \mathrm{L}$ of S.O.C. medium was added to each vial. The vials were tightly capped and shaken horizontally at $37^{\circ} \mathrm{C}$ for $1 \mathrm{~h}$ at $220 \mathrm{rpm}$ in a shaking incubator. Then, $100 \mu \mathrm{L}$ of the transformation mixture was uniformly spread using a lazy-L spreader on a pre-warmed Luria-Bertani (LB) agar plate $(\mathrm{Amp}+)$. The plates were incubated at $37{ }^{\circ} \mathrm{C}$ overnight, after which visible colonies appeared. A single colony of transformed bacteria was inoculated and grown overnight in $2 \mathrm{~mL}$ of pre-warmed LB medium in a shaking incubator at $220 \mathrm{rpm}$. To obtain the highest possible plasmid concentration, four individual colonies were chosen. Commercially available PureYield ${ }^{\mathrm{TM}}$ Plasmid Miniprep/Maxiprep kits (Promega, Madison, WI, USA) were used to isolate and amplify the plasmids.

\section{White-opsin synthesis}

MultiSite Gateway® Technology ${ }^{26}$ was used to fuse multiple opsinencoding genes (ChR2, C1V1, ReaChR) to create White-opsin (Supplementary Fig. S1). In the 4-fragment recombination reaction, four PCR products (promoter, ChR2, C1V1, and ReaChR) flanked by specific attB or attBr sites and four donor vectors were used in separate $\mathrm{BP}$ recombination reactions to generate four entry clones. These four entry clones and the destination vector, $\mathrm{pDEST}^{\mathrm{TM}} \mathrm{R} 4-\mathrm{R} 3$ Vector II, were used together in an LR recombination reaction to create the White-opsin construct.

\section{DNA gel electrophoresis}

To verify the opsin-plasmid constructs, DNA gel electrophoresis of the plasmids (with restriction enzymes) and a known DNA ladder (as a reference) was performed. First, $1 \mathrm{~g}$ of agarose was weighed and mixed with $100 \mathrm{~mL}$ of $1 \times$ Tris-acetate-EDTA (TAE) buffer. The solution was placed in a microwave for 1-3 min until the agarose was completely dissolved. The agarose solution was allowed to cool for $5 \mathrm{~min}$. Ethidium bromide (EtBr) was then added to a final concentration of approximately $0.2 \mu \mathrm{g} \mathrm{mL} \mathrm{m}^{-1}$. The agarose gel was poured into a gel tray with a well comb in place. It was allowed to sit at room temperature until the gel solidified, and the agarose gel was then loaded into the gel box (electrophoresis unit). The gel box was filled with $1 \times \mathrm{TAE}$ until the gel was immersed. Restriction enzymes (Fermentas, MA, USA) were used to digest the plasmids. The DNA ladder (Promega) and the opsin plasmids (ChR2, ReaChR and White-opsin) were loaded into different lanes, and the gel was run at $100 \mathrm{~V}$ until the dye line was approximately $75 \%$ of the way down the gel. The power supply was then switched off, and the gel was carefully removed and placed under UV light for imaging (Gel Logic 212 PRO). The sizes of the bands (reference: DNA ladder) were found to match the expected sizes (Supplementary Fig. S1d). After the verification of proper band size, $5 \mathrm{~L}$ of bacteria were cultured to produce a large volume of plasmids using the PureYield Maxiprep kit (Promega). The concentration of the amplified plasmids was measured using a spectrophotometer (ND-1000, Nano Drop), and the results are shown in Supplementary Fig. S1e-S1g.

\section{Cell culture}

HEK293 cells were transfected with ChR2, ReaChR or the White-opsin construct using Lipofectamine (Life Technologies, Grand Island, NY, USA). $24 \mathrm{~h}$ after transfection, the HEK293 cells were cultured in Petri dishes and maintained in Dulbecco's modified Eagle's medium (DMEM)/F-12 with $10 \%$ fetal bovine serum, $0.2 \mathrm{mg} \mathrm{mL}^{-1}$ streptomycin, and $200 \mathrm{U} \mathrm{mL}^{-1}$ penicillin. The cultures were maintained at $37^{\circ} \mathrm{C}$ in a humidified $5 \% \mathrm{CO}_{2}$ atmosphere. For optoporation of the opsin plasmids, the cells were plated on poly-D-lysine-coated glass-bottomed $35 \mathrm{~mm}$ Petri dishes (MatTek Corporation, MA, USA). Visualization of the reporter (YFP or Citrine) fluorescence under suitable illumination allowed the identification of transgene-expressing cells. The cells were incubated with all-trans-retinal (ATR, $1 \mu \mathrm{M}$ ) for at least $6 \mathrm{~h}$ before the patch-clamp experiments were performed.

\section{Setup for optoporation}

A schematic diagram of the experimental setup for optoporation is shown in Figure 1a. A beam (pulse width: $\sim 100 \mathrm{fs}$ ) from a tunable Ti:sapphire laser (L1, Newport Spectra-Physics, Inc., Santa Clara, CA, USA) was expanded (by a beam expander, BE) and directed toward the sample by a dichroic mirror (DM1) through an inverted microscope (Nikon, Ti:U Eclipse). A $100 \times$ (numerical aperture $(\mathrm{NA})=1.4$ ) microscope objective was used to focus the laser beam to a diffraction-limited spot (Figure 1b) on the top surface of the cell. The focal plane of the laser was adjusted to match the imaging plane. Excitation (Ex) and emission (Em) filters were used to transmit and collect the appropriate bands of visible light to and from the sample and to block any remaining backscattered laser light. A second dichroic mirror (DM2) was used to reflect the fluorescence excitation light from the mercury lamp (FL). The images were acquired by a cooled EMCCD (Cascade 1K, Photometrics, Tucson, AZ, USA) and processed using ImageJ software (NIH). The number of fs laser pulses irradiating each cell was controlled by an external electromechanical shutter ( $\mathrm{S}$, Uniblitz, Vincent Associates, NY, USA). The laser beam power at the sample site was controlled by the laser (Mai Tai) interface software through alterations to the orientation of the polarizer $(\mathrm{P})$. The average beam power at the sample site was calculated by multiplying the transmission factor of the microscope objective ${ }^{27}$ by the average power 


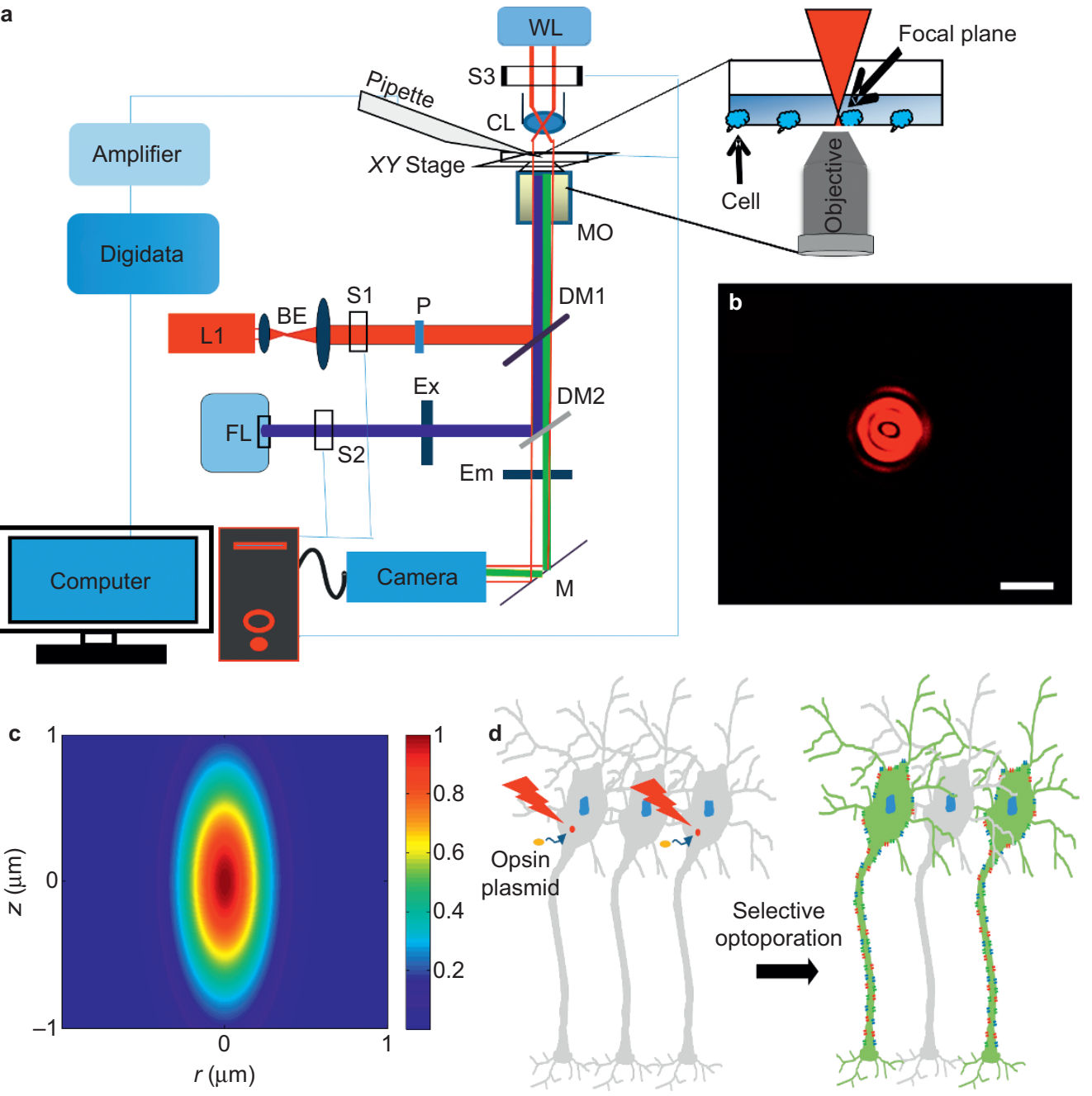

Figure 1 Targeted ultrafast laser-assisted opsin delivery setup and beam parameters. (a) Optoporation setup. L1, Ti:sapphire laser; BE, beam expander; S1-S3, shutters; P, polarizer; FL, fluorescence excitation; Ex/Em, excitation/emission filters; MO, microscope objective; WL, white-light source; CL, condenser lens; DM1 and DM2, dichroic mirrors; M, mirror. (b) Laser spot measured at the focal plane. Scale bar: $10 \mu \mathrm{m}$. (c) Intensity distribution in the XZ plane in the focal volume (wavelength: $850 \mathrm{~nm}$, focused by an objective with NA = 1.4). (d) Schematic illustration of the optical delivery of opsin-encoding plasmids to target cells (lightning symbol) leading to selective opsin expression (detected by a fluorescent reporter protein).

measured at the back aperture of the objective. The laser parameters (energy per pulse and exposure time) were optimized (pulse energy: $1 \mathrm{~nJ}$, exposure time: $20 \mathrm{~ms}$, repetition rate: $76 \mathrm{MHz}$ ) and held constant in all experiments. Gridded glass-bottomed Petri dishes (MatTek, Ashland, MA, USA) were used to locate the exact positions of optoporation. In certain cases, when the cells were in a monolayer and the grid could not be visualized, a permanent marking pen with a sharp tip was used to mark small circles (radius: $\sim 0.3 \mathrm{~mm}$ ) on the bottom of the glass Petri dish, and a diamond marker was used to mark a cross within each circle.

\section{Opsin expression and optogenetic stimulation}

The expression of the opsins was observed after $36 \mathrm{~h}$ of transfection (lipofection or optoporation) via epifluorescence microscopy of the reporter proteins using a $20 \times$ or $100 \times$ microscope objective. The onetailed $t$-test was used to determine the statistical significance of the difference in fluorescence expression. Broadband white light from a halogen lamp was coupled to a multimode optical fiber for optogenetic stimulation. A power meter (PM 100D, Thorlabs, Inc., NJ, USA) was used to measure the light power at the sample plane. An electromechanical shutter was used to control the duration of the stimulation pulses. The shutter was synchronized with the patch-clamp recording system (Molecular Devices, Sunnyvale, CA, USA).

\section{Opto-electrophysiology setup}

The opto-electrophysiology setup consisted of an upright fluorescence microscope (Olympus, BX 60M) and an amplifier system (Axon Multiclamp 700B, Molecular Devices). For whole-cell patch-clamp experiments, borosilicate micropipettes with resistances of 3-4 M $\Omega$ were prepared using a pipette puller (Sutter Instruments, Novato, CA, USA). Each micropipette was filled with a solution of $130 \mathrm{mM} \mathrm{K}$-gluconate, $7 \mathrm{mM} \mathrm{KCl,} 2 \mathrm{mM} \mathrm{NaCl}, 1 \mathrm{mM}$ $\mathrm{MgCl}_{2}, 0.4 \mathrm{mM}$ EGTA, $10 \mathrm{mM}$ HEPES, $2 \mathrm{mM}$ ATP-Mg, $0.3 \mathrm{mM}$ GTP-Tris, and $20 \mathrm{mM}$ sucrose. The electrode was mounted on a motorized micromanipulator (Sutter Instruments, MP225). The standard extracellular solution of $150 \mathrm{mM} \mathrm{NaCl}, 10 \mathrm{mM}$ glucose, $5 \mathrm{mM} \mathrm{KCl}, 2 \mathrm{mM} \mathrm{CaCl}_{2}$, and $1 \mathrm{mM} \mathrm{MgCl}_{2}$ was buffered with 10 mM HEPES ( $\mathrm{pH}$ 7.3). The patched cells were maintained at -70 
$\mathrm{mV}$, and the output from the amplifier was digitized using a Digidata 1440 instrument (Molecular Devices). For the patchclamp measurements, the hardware was interfaced with patchclamp software (pClamp 10, Molecular Devices). For activation of the opsin-expressing cells, the stimulation beam was delivered by a liquid light guide. The electromechanical shutter in the light path was interfaced with a PC for generation and control of the light pulses for optogenetic stimulation. The patch-clamp recording system was electrically isolated using a Faraday cage. pClamp 10 software (Molecular Devices) was used for patch-clamp data analysis.

\section{RESULTS AND DISCUSSION}

Transfection of a single opsin and multiple opsins via lipofection To evaluate the transfection of mammalian cells with plasmids encoding either a single opsin or multiple opsins via lipofection, the molecular engineering of single and multiple opsins (a fusion of ChR2, C1V1, and ReaChR named 'White-opsin') was performed. Supplementary Fig. S1 shows vector maps of ChR2 (a), ReaChR (b), and Whiteopsin (c). The corresponding gel electrophoresis images of each opsin are shown in Supplementary Fig. S1d. HEK293 cells were transfected using Lipofectamine and plasmids at a final concentration of $1 \mu \mathrm{g} \mathrm{mL}^{-1}$, as measured by the absorption spectra of cloned ChR2, ReaChR, and White-opsin (Supplementary Fig. S1e-S1g). The quantification results for the Lipofectamine-based transfection of the different opsins are shown in Figure 2. In Figure 2a and 2b, we show the representative bright-field and fluorescence images of HEK293 cells transfected with ChR2-YFP using Lipofectamine. To quantify expression in the plasma membrane and the intracellular components, lines were drawn across representative cells, and the fluorescence intensity profiles of the opsin-reporter protein (YFP) along these lines were plotted (Figure 2c). A quantitative comparison of the expression of ChR2 in the membrane and intracellular components is shown in Figure 2d. Similarly, the expression levels of ReaChR (Figure 2e-2h) and White-opsin (Figure 2i-2l) after transfection via lipofection were quantified. Regardless of the type of opsin, the cellular morphology was observed to be compromised, changing from a flat to a spherical shape. This resulted in higher intracellular fluorescence, leading to the absence of any distinct peaks (across the membrane), as seen in Figure $2 \mathrm{c}, 2 \mathrm{~g}$, and $2 \mathrm{k}$. Therefore, no significant differences between membrane and intracellular expression were observed (Figure 2d, 2h, and 2l).

\section{Targeted laser-assisted transfection of single opsins}

To achieve the targeted transfection of cells with opsin-encoding plasmids (ChR2 and ReaChR), we used optimized laser parameters ${ }^{23}$ for optoporation into HEK293 cells. Before optoporation, the extracellular medium was exchanged with new DMEM medium-containing DNA plasmids (ReaChR or ChR2) with a final concentration of $1 \mu \mathrm{g} \mathrm{mL}^{-1}$ and incubated for $30 \mathrm{~min}$. Figure la shows the setup for targeted ultrafast laser-assisted opsin delivery. The focused laser spot is shown in Figure 1b; the simulated intensity distribution of the spot in the XZ plane in the focal volume (wavelength: $850 \mathrm{~nm}$, focused by an objective with NA $=1.4$ ) is presented as Figure 1c. Using this localized focal volume, spatially targeted cells can be selectively optoporated, as schematically illustrated in Figure 1d. Figure 3 shows the targeted fs laser-assisted transfection of single opsins into HEK293 cells. A representative fluorescence image of targeted ChR2-YFP-optoporated HEK293 cells (Figure 3a) is overlaid on the corresponding bright-field image (Figure $3 \mathrm{~b}$ ). Similarly, Figure $3 \mathrm{c}$ and $3 \mathrm{~d}$ shows representative laser-targeted ReaChR-transfected HEK293 cells. Unlike in the case of
Lipofectamine-based transfection, the cellular morphology after laser transfection was found to remain flat. The fluorescence intensity profiles of the opsin-reporter proteins (YFP and Citrine) along the lines drawn across representative cells (Figure $3 \mathrm{e}$ and $3 \mathrm{f}$ ) exhibit distinct peaks at the plasma membranes, implying the localized expression of ChR2 and ReaChR on the plasma membrane (Figure $3 \mathrm{~g}$ and $3 \mathrm{~h}$ ). Figure $4 \mathrm{e}$ shows a quantitative comparison between the ChR2 and ReaChR expression distributions (ratio of membrane to intracellular expression) after optoporation and lipofection.

\section{Laser-assisted transfection of White-opsin: functional evaluation using broadband light}

Figure 4 shows the targeted laser-assisted transfection of HEK293 cells with White-opsin (fusion of multiple opsins) and the subsequent broadband (white)-light-based optogenetic stimulation. Visualizations of the transfection of HEK293 cells with White-opsin plasmids via optoporation or lipofection are shown in Supplementary Fig. S2. An increase in intracellular fluorescence and a few distinct specks (attributed to the propidium iodide staining of White-opsin plasmids) after optoporation (at the site marked by an arrow in Supplementary Fig. S2a) were observed $1 \mathrm{~min}$ after optoporation (Supplementary Fig. S2c). The transfected plasmid(s) were transcribed and translated into proteins (i.e., White-opsin and the reporter YFP), which were expressed in the targeted organelle (i.e., the plasma membrane). Similar to the ChR2- and ReaChR-optoporated cells, the White-opsin delivered by the fs laser was found to be preferentially expressed on the plasma membrane (Figure 4a). The non-optoporated (control) cells were observed not to express the fluorescent reporter protein. Figure $4 \mathrm{~b}$ shows the representative fluorescence intensity profiles of the reporter proteins along lines drawn across cells (e.g., the dotted white line in Figure 4a). A quantitative comparison of the expression in the membranes and intracellular components of cells optoporated with White-opsin is shown in Figure 4c. To evaluate the functional activation of White-opsin, broadband stimulation was applied to the White-opsin-expressing cells and patch-clamp electrophysiology was used to record the photoinduced currents. Representative inward photocurrents generated in response to white-light optogenetic stimulation $\left(0.12 \mathrm{~mW} \mathrm{~mm}^{-2}\right)$ at two different pulse widths are shown in Figure 4d; these results suggest that lowintensity broadband light can be used to generate a robust photocurrent in a spatially targeted manner with high temporal resolution. The white-light-induced photocurrent generated in the Whiteopsin-expressing cells (Figure 4d) was significantly higher than the photocurrent in cells expressing ChR2 (Supplementary Fig. S3).

\section{Quantitative comparison between two non-viral gene delivery methods}

Calculation of the total intensities from individual cells revealed no significant differences between lipofection and optoporation. Figure $4 \mathrm{e}$ summarizes the quantitative comparison between the different opsin expression distributions (ratios of membrane to intracellular expression) resulting from the two different non-viral gene delivery methods. The higher membrane-to-intracellular ratio of expression in optoporated cells than lipofected cells (Figure 4e) can be attributed to the flat morphology of the optoporated opsinexpressing cells compared with those transfected via lipofection, which tended to become spherical. This alteration in the morphology of lipofected cells was confirmed by three-dimensional confocal microscopy (Supplementary Fig. S4). 

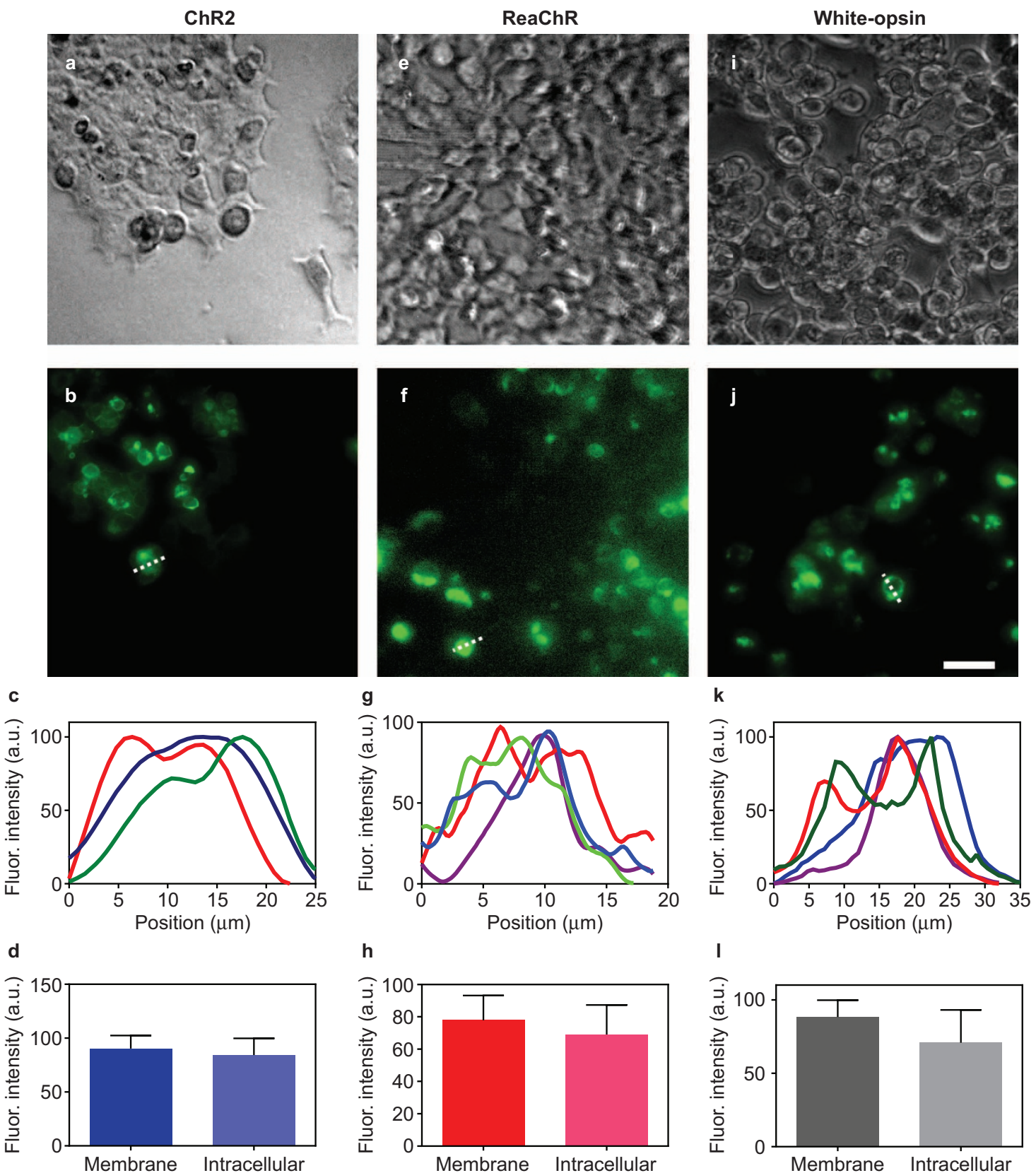

Figure 2 Quantification results for the Lipofectamine-based transfection of different opsins. (a-d) ChR2-, (e-h) ReaChR-, and (i-I) White-opsin-transfected HEK293 cells. (a, e, i) Bright-field and (b, f, j) fluorescence images of HEK293 cells transfected with ChR2-YFP, ReaChR, and White-opsin, respectively, imaged using a 20X objective. Scale bar: $20 \mu \mathrm{m}$. (c, g, k) Representative intensity profiles of the opsin-reporter protein fluorescence along the lines drawn across the cells. (d, $\mathbf{h}, \mathbf{I})$ Quantitative comparison of expression in membrane and intracellular components in ChR2-, ReaChR-, and White-opsin-transfected cells. $N=6 / o p s i n$. Average \pm S.D. No significant difference is observed between the membrane and the intracellular components. Flour., fluorescence.

Although the use of opsins to modulate cellular activity has been rapidly increasing, the existing methods (e.g., virus-mediated approaches and lipofection) of delivering those opsins lack the ability to achieve targeted expression. Traditional methods of targeted delivery of impermeable molecules into cells require microinjection using a mechanical needle or microelectrodes, which often causes cell death and compromises sterility. Carbon ${ }^{28,29}$ and gold ${ }^{30,31}$ nanoparticles in conjunction with pulsed ${ }^{28}$ or continuous-wave ${ }^{29}$ laser beams have been employed for the delivery of exogenous small molecules to cells; this approach requires the presence of the nano- particles along with the molecules to be delivered in the vicinity of the membrane of the target cell. Furthermore, in viral delivery methods, the size of the plasmids that can be packaged for delivery is limited, which may restrict the use of these methods to the delivery of only single-opsin plasmids rather than multiple opsins (such as White-opsin). The optoporation of one or more opsin plasmids into cells offers significant advantages over other methods of delivery (e.g., micro-injection, electroporation, lipofection, and viral transfection) because this method provides the ability to deliver large therapeutic molecules (e.g., $>10 \mathrm{~kb}$ ), spatial and temporal 

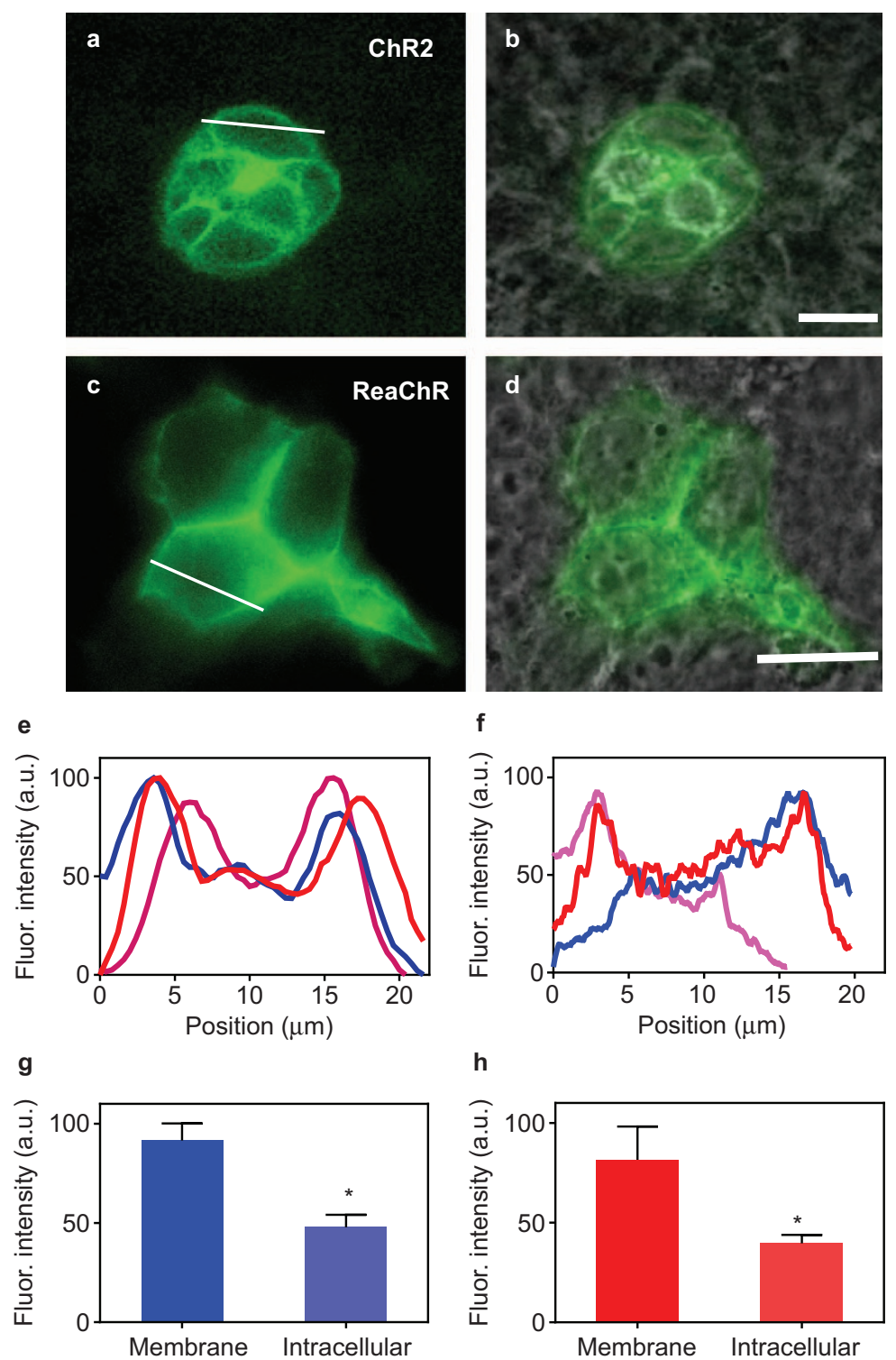

Figure 3 Targeted laser-assisted transfection of single opsins. (a) Representative fluorescence image of targeted ChR2-YFP-optoporated HEK293 cells, (b) overlaid on the corresponding bright-field image showing highly packed cells in a monolayer, imaged using a $20 \times$ objective. (c) Representative fluorescence image of targeted HEK293 cells optoporated with ReaChR, (d) overlaid on the corresponding bright-field image showing cells in a monolayer, imaged using a 100× objective. Scale bar: $15 \mu \mathrm{m}$. (e, f) Representative fluorescence intensity profiles of the reporter proteins along the lines drawn across the cells (white lines in $\mathbf{a}, \mathbf{c}$ ). (g, $\mathbf{h}$ ) Quantitative comparison of expression in the membrane and the intracellular components in cells optoporated with ChR2 and ReaChR. $N=5 /$ opsin. Average \pm S.D. ${ }^{*} p<0.01$ difference between the membranes and intracellular components of opsin-expressing cells. Flour., fluorescence.

selectivity, and absolute sterility. Furthermore, it can be used for the delivery of multiple-opsin plasmids into a single cell or for the delivery of single-opsin plasmids into multiple cells through multiplexing of the laser beam.

A substantial barrier to the clinical translation of optogenetic methods of vision restoration is the delivery of opsin-encoding genes into spatially targeted regions of a retina that has degenerated. For example, the spatially targeted delivery of opsin-encoding genes to either the peripheral retina or macula, which will begin to lose light sensitivity through the loss of photoreceptors, is required in cases of RP and AMD, respectively. Furthermore, patient-to-patient variability and time-dependent changes in the spatial distribution of retinal degeneration demand the site-specific expression of opsins. Unlike viral methods, the use of spatially targeted optoporation will enable the expression of one or more opsins in areas of retinal degeneration, thereby allowing for effective vision restoration. Using viral or other non-viral (e.g., electroporation and lipofection) methods, the constructs will be delivered universally, inducing uncontrolled expression over the entire retina and perturbing the already functioning retinal regions. Furthermore, the delivery of macro-constructs encoding multiple opsins with spectrally separated activation peaks over the entire visible spectrum will lead to higher photocurrents in response to ambient white light. This will enable the generation of action potentials in White-opsin-sensitized higher-order neurons at a much lower spectral density of intensity (intensity/bandwidth) than can be achieved through the conventional use of monochromatic light and single narrow-band opsins. Although different viral vectors can separately deliver different plasmids encoding for opsins for the 


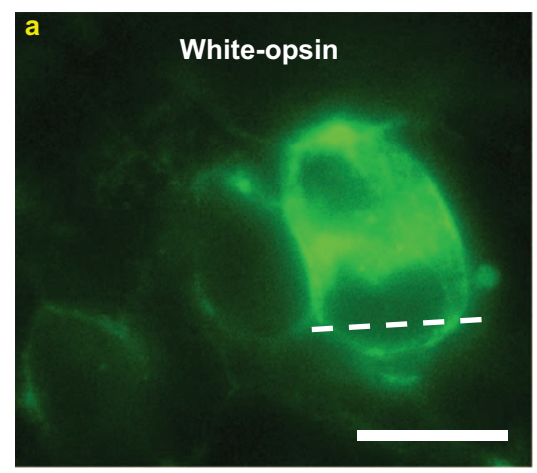

b
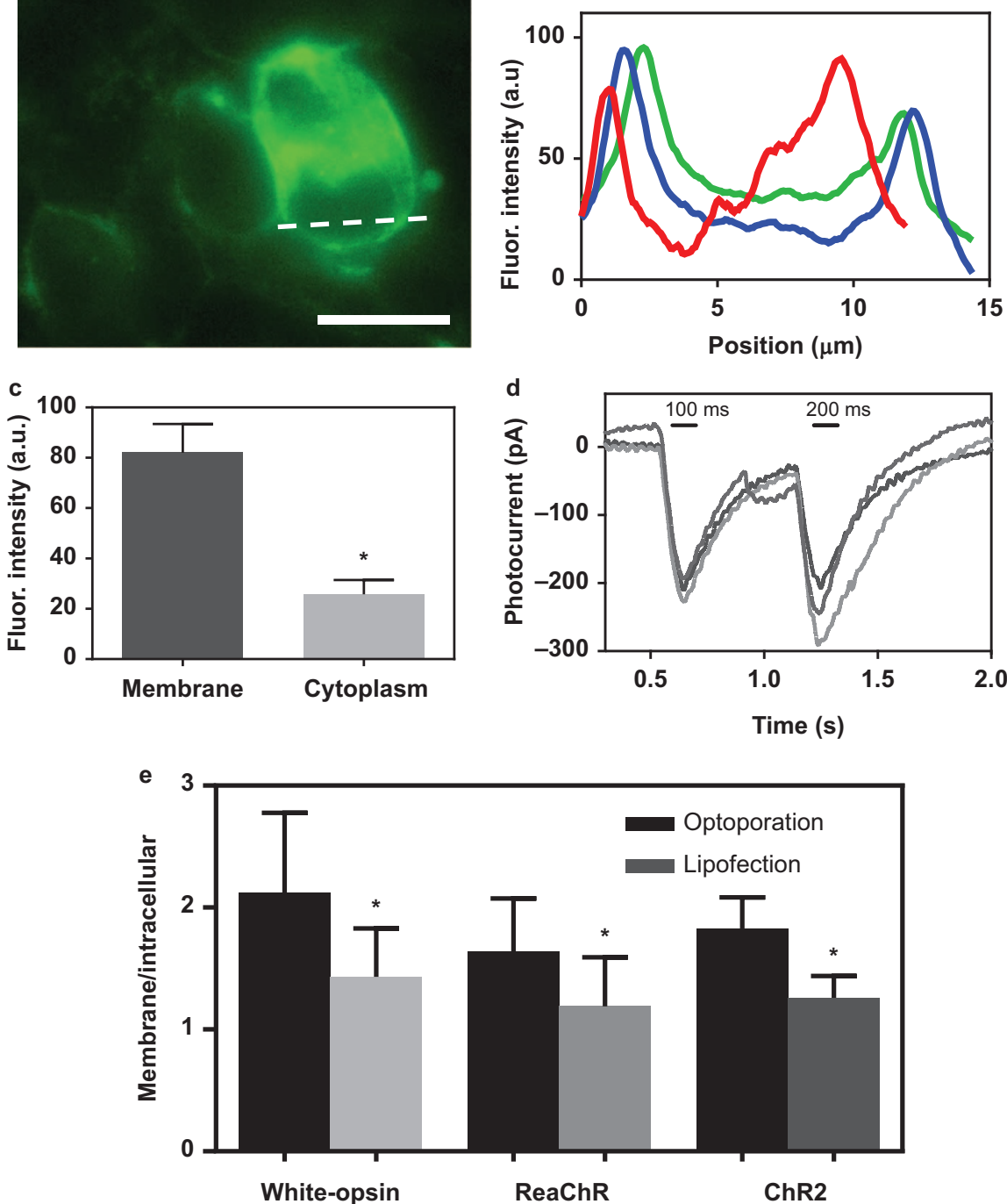

Figure 4 Targeted laser-assisted transfection of multiple opsins: functional evaluation and quantitative comparison between two non-viral delivery methods. (a) Fluorescence image of HEK293 cells after optoporation with White-opsin, imaged using a 100× objective. Scale bar: $15 \mu \mathrm{m}$. (b) Representative fluorescence intensity profiles of reporter proteins along lines drawn across cells (e.g., the dotted white line in a). (c) Quantitative comparison of the expression in the membranes and intracellular components of cells optoporated with White-opsin. $N=5$. Average \pm S.D. $* p<0.01$ difference between the membranes and intracellular components of White-opsinexpressing cells. (d) Representative inward photocurrent profiles in response to white-light optogenetic stimulation $\left(0.12 \mathrm{~mW} \mathrm{~mm}^{-2}\right)$ at two different pulse widths (100 and $200 \mathrm{~ms}$ ). (e) Quantitative comparison between the opsin expression distributions (ratios of membrane to intracellular expression) observed after optoporation and

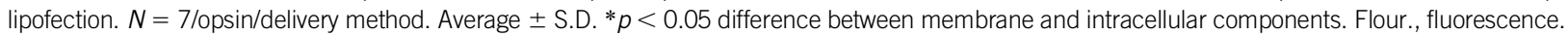

different spectral components, identical stoichiometric expressions of the different spectrally sensitive components in each cell cannot be guaranteed. Therefore, we believe that the application of the spatially targeted laser transfection of macro-constructs such as White-opsin into a retina that has degenerated will allow the efficient stimulation of higher-order retinal cells in areas of photoreceptor degeneration with ambient white light, leading to visual restoration.

The surgical risks of existing complex retinal-implantation procedures for improving vision are very high. This is a particular concern when an implant must be replaced because of device failure during chronic usage. Our method of laser-based targeted delivery of opsins is easy to incorporate into current clinical practice via the intravitreal injection of multiple-opsin-encoding plasmids followed by the ultrafast NIR laser-based non-contact creation of highly localized, transient pores on the outer RGC membrane in photodegenerated areas. A reasonable delivery time of $\sim 45 \mathrm{~min}$ for an area containing 500 000 cells is expected for $5 \mathrm{~ms}$ of exposure per cell. Furthermore, in the case of loss of opsin expression or the degeneration of new retinal area(s), the entire process can be easily repeated. For in vitro or small animal models, this method can be practiced using any commercial multiphoton microscope, and the same system can be used to both transfect and stimulate (via two-photon stimulation ${ }^{10}$ ) opsin-sensitized cells with high spatial accuracy.

\section{CONCLUSIONS}

To conclude, an ultrafast NIR laser microbeam was used to deliver both single opsins and large multi-opsin constructs to target cells. The effectiveness and function of the multiple-opsin-sensitized cells were 
confirmed via patch-clamp electrophysiology. The NIR optoporation method will eliminate the requirement for multiple virus delivery and the possible immunogenic reactions, as well as offering the ability to deliver macro-constructs encoding opsins that are sensitive to a broad spectral range to spatially targeted areas of retinal degeneration. Such spatially resolved delivery of genes to the peripheral retina/macula is ideal for sensitizing retinal areas that have degenerated, thus paving the way toward restoring lost vision in RP/AMD patients. This will allow a higher sensitivity of opsin-sensitized higher-order neurons in targeted retinal areas (those suffering from photodegeneration) to ambient white light and, therefore, will significantly lower the activation threshold compared with the conventional approaches based on narrow-band opsins and/or active stimulation based on intense narrow-band light.

\section{CONFLICT OF INTEREST}

Dr. Samarendra Mohanty is a founder of, and has an equity interest in NanoScope Technologies, LLC, which is developing products in Biomedical diagnostics and therapeutic technologies. The terms of this arrangement have been reviewed and approved by the University of Texas at Arlington in accordance with its policy on objectivity in research.

\section{ACKNOWLEDGEMENTS}

The authors would like to thank Christopher Cote, Gregory Cervenka, Prof. Qing Lin, Saurabh Kokane, and Sarmishtha Satpathy (UTA) for their assistance with the experiments. Samarendra Mohanty would like to thank K. Deisseroth (Stanford) for the ChR2 and C1V1 plasmids and J. Lin (UCSD) for the ReaChR construct. Samarendra Mohanty would also like to acknowledge the support from the National Institute of Health (NS084311) and the Office of the President and Provost of The University of Texas at Arlington.

1 Bi AD, Cui JJ, Ma YP, Olshevskaya E, Pu ML et al. Ectopic expression of a microbialtype rhodopsin restores visual responses in mice with photoreceptor degeneration. Neuron 2006; 50: 23-33.

2 Thyagarajan S, van Wyk M, Lehmann K, Löwel S, Feng GP et al. Visual function in mice with photoreceptor degeneration and transgenic expression of channelrhodopsin 2 in ganglion cells. J Neurosci 2010; 30: 8745-8758.

3 Zhang Y, Ivanova E, Bi AD, Pan ZH. Ectopic expression of multiple microbial rhodopsins restores $\mathrm{ON}$ and OFF light responses in retinas with photoreceptor degeneration. J Neurosci 2009; 29: 9186-9196.

4 Tomita H, Sugano E, Isago H, Hiroi T, Wang Z et al. Channelrhodopsin-2 gene transduced into retinal ganglion cells restores functional vision in genetically blind rats. Exp Eye Res 2010; 90: 429-436.

5 Lagali PS, Balya D, Awatramani GB, Münch TA, Kim DS et al. Light-activated channels targeted to $\mathrm{ON}$ bipolar cells restore visual function in retinal degeneration. Nat Neurosci 2008; 11: 667-675.

6 Busskamp V, Duebel J, Balya D, Fradot M, Viney TJ et al. Genetic reactivation of cone photoreceptors restores visual responses in retinitis pigmentosa. Science 2010; 329: 413-417.

7 Boyden ES, Zhang F, Bamberg E, Nagel G, Deisseroth K. Millisecond-timescale, genetically targeted optical control of neural activity. Nat Neurosci 2005; 8: 12631268.

8 Nagel G, Szellas T, Huhn W, Kateriya S, Adeishvili N et al. Channelrhodopsin-2, a directly light-gated cation-selective membrane channel. Proc Natl Acad Sci USA 2003; 100: 13940-13945.
9 Cao H, Gu L, Mohanty SK, Chiao JJ. An Integrated $\mu$ LED Optrode for Optogenetic Stimulation and Electrical Recording. IEEE Trans Biomed Eng 2013; 60: 225-229.

10 Dhakal KR, Gu L, Shivalingaiah S, Dennis TS, Morris-Bobzean SA et al. Non-scanning fiber-optic near-infrared beam led to two-photon optogenetic stimulation in-vivo. PLOS ONE 2014; 9: e111488.

11 Zhang F, Aravanis AM, Adamantidis A, de Lecea L, Deisseroth K. Circuit-breakers: optical technologies for probing neural signals and systems. Nat Rev Neurosci 2007 8: 577-581.

12 Mohanty SK, Reinscheid RK, Liu X, Okamura N, Krasieva TB et al. In-depth activation of Channelrhodopsin 2-sensitized excitable cells with high spatial resolution using two-photon excitation with a near-infrared laser microbeam. Biophys J 2008; 95 : 3916-3926.

13 Satpathy S, Batabyal S, Dhakal KR, Lin J, Kim Y et al. Broad spectral excitation of opsin for enhanced stimulation of cells. Opt Lett 2015; 40: 2465-2468.

14 Mohanty SK, Lakshminarayananan V. Optical techniques in optogenetics. J Modern Optics 2015; 62: 949-970.

15 Tirlapur UK, König K. Targeted transfection by femtosecond laser. Nature 2002; 418: 290-291.

16 Soman P, Zhang WD, Umeda A, Zhang ZJ, Chen SC. Femtosecond laser-assisted optoporation for drug and gene delivery into single mammalian cells. J Biomed Nanotechnol 2011; 7: 334-341.

17 Stracke F, Rieman I, König K. Optical nanoinjection of macromolecules into vital cells. J Photoch Photobio B 2005; 81: 136-142.

18 Barrett LE, Sul JY, Takano H, van Bockstaele EJ, Haydon PG et al. Region-directed phototransfection reveals the functional significance of a dendritically synthesized transcription factor. Nat Methods 2006; 3: 455-460.

19 Peng C, Palazzo RE, Wilke I. Laser intensity dependence of femtosecond near-infrared optoinjection. Phys Rev E 2007; 75: 041903.

20 Kohli V, Elezzabi AY. Laser surgery of zebrafish (Danio rerio) embryos using femtosecond laser pulses: optimal parameters for exogenous material delivery, and the laser's effect on short- and long-term development. BMC Biotechnol 2008; 8: 7 .

21 Lei M, Xu HP, Yang H, Yao BL. Femtosecond laser-assisted microinjection into living neurons. J Neurosci Meth 2008; 174: 215-218.

22 Uchugonova A, König K, Bueckle R, Isemann A, Tempea G. Targeted transfection of stem cells with sub-20 femtosecond laser pulses. Opt Express 2008; 16: 9357 9364.

23 Dhakal K, Black B, Mohanty S. Introduction of impermeable actin-staining molecules to mammalian cells by optoporation. Sci Rep 2014; 4: 6553.

24 Zeira E, Manevitch A, Khatchatouriants A, Pappo O, Hyam E et al. Femtosecond infrared laser - an efficient and safe in vivo gene delivery system for prolonged expression. Mol Ther 2003; 8: 342-350.

25 Gu L, Mohanty SK. Targeted microinjection into cells and retina using optoporation. J Biomed Opt 2011; 16: 128003.

26 Buj R, Iglesias N, Planas AM, Santalucía T. A plasmid toolkit for cloning chimeric CDNAs encoding customized fusion proteins into any Gateway destination expression vector. BMC Mol Biol 2013; 14: 18.

27 Kong X, Mohanty SK, Stephens J, Heale JT, Gomez-Godinez V et al. Comparative analysis of different laser systems to study cellular responses to DNA damage in mammalian cells. Nucleic Acids Res 2009; 37: e68.

28 Chakravarty P, Qian W, El-Sayed MA, Prausnitz MR. Delivery of molecules into cells using carbon nanoparticles activated by femtosecond laser pulses. Nat Nanotechnol 2010; 5: 607-611.

29 Gu L, Koymen AR, Mohanty SK. Crystalline magnetic carbon nanoparticle assisted photothermal delivery into cells using CW near-infrared laser beam. Sci Rep 2014; 4: 5106.

30 Xiong RH, Raemdonck K, Peynshaert K, Lentacker I, de Cock I et al. Comparison of gold nanoparticle mediated photoporation: vapor nanobubbles outperform direct heating for delivering macromolecules in live cells. Acs Nano 2014; 8: 6288-6296.

31 Lukianova-Hleb EY, Mutonga MBG, Lapotko DO Cell-specific multifunctional processing of heterogeneous cell systems in a single laser pulse treatment. ACS Nano 2012; 6: 10973-10981.

This work is licensed under a Creative Commons AttributionNonCommercial-NoDerivs 4.0 Unported License. The images or other third party material in this article are included in the article's Creative Commons license, unless indicated otherwise in the credit line; if the material is not included under the Creative Commons license, users will need to obtain permission from the license holder to reproduce the material. To view a copy of this license, visit http://creativecommons.org/licenses/ by-nc-nd/4.0/ 